\title{
Dynamic Wireless Network Reconfiguration for Control System applied to a Nuclear Reactor Case Study
}

\author{
Wenchen Wang, Daniel Mosse \\ Real-time Systems Lab \\ Computer Science Department \\ University of Pittsburgh
}

\author{
Daniel Cole \\ Nuclear Engineering Research Lab \\ Mechanical Eng and Materials \\ Science Department \\ University of Pittsburgh
}

\author{
Jason G. Pickel \\ Algorithm Engineer \\ Automated Driving \\ Zenuity
}

\begin{abstract}
Control systems using sensors and wireless networks are becoming more prevalent, due to its ease of deployment (no wires and no electricity) and maintenance (longer lifetime). However, interference and noise can cause delays and packet losses that can degrade control system performance, which leads us to find the optimal network configuration to minimize that impact. Another problem of wireless networks for control systems is caused by time-varying fault patterns, which motivates us to do network reconfiguration at runtime. We focus on the online wireless network reconfiguration in an embedded networked and cyber-physical system (CPS), with four main contributions: (1) the design and implementation of a novel network reconfiguration framework with offline and online components that consider time-correlated link failures; (2) a network imperfection model; (3) six online reconfiguration algorithms for wireless control system; (4) a case study with 12 hops and up to 50 nodes that controls a nonlinear small modular nuclear reactor. Our network imperfection model is accurate (with 0.993 Pearson correlation) and our online reconfiguration algorithms have smaller error and longer network lifetime than a state-of-theart static scheme.
\end{abstract}

\section{CCS CONCEPTS}

- Computer systems organization $\rightarrow$ Embedded systems; Redundancy; Robotics; • Networks $\rightarrow$ Network reliability;

\section{KEYWORDS}

wireless control system, wireless network reconfiguration, CPS

\section{ACM Reference Format:}

Wenchen Wang, Daniel Mosse, Daniel Cole, and Jason G. Pickel. 2018. Dynamic Wireless Network Reconfiguration for Control System applied to a Nuclear Reactor Case Study. In 26th International Conference on Real-Time Networks and Systems (RTNS '18), October 10-12, 2018, Chasseneuil-du-Poitou, France. ACM, New York, NY, USA, 11 pages. https://doi.org/10.1145/3273905. 3273912

Work performed under grants: DOE DE-NE000739, NSF 15-35232 and 13-05220.

Permission to make digital or hard copies of all or part of this work for personal or classroom use is granted without fee provided that copies are not made or distributed for profit or commercial advantage and that copies bear this notice and the full citation on the first page. Copyrights for components of this work owned by others than the author(s) must be honored. Abstracting with credit is permitted. To copy otherwise, or republish, to post on servers or to redistribute to lists, requires prior specific permission and/or a fee. Request permissions from permissions@acm.org.

RTNS '18, October 10-12, 2018, Chasseneuil-du-Poitou, France

๑ 2018 Copyright held by the owner/author(s). Publication rights licensed to ACM. ACM ISBN $978-1-4503-6463-8 / 18 / 10 \ldots \$ 15.00$

https://doi.org/10.1145/3273905.3273912

\section{INTRODUCTION}

Wireless control systems (WCS) are composed of controllers, sensors and actuators connected via a multi-hop wireless network [7, $12,15,16,25,26,34]$. Given that the control room is usually geographically distant from the sensors and actuators, wireless networks are good due to the lack of wires (electrical or networking). However, wireless network delays to/from the control room and packet losses can induce undesirable errors in the control system. For example, in a nuclear power plant (NPP), there could be the loss of efficiency, wasting megawatts of power. We focus on these network-induced imperfections [40]: time delays and packet losses.

In practice, the control sampling period (i.e., the interval where the control loop makes decisions) depends on the plant being controlled, and typically varies from $250 \mathrm{~ms}$ to 8 minutes [7]. For each cyberphysical system (CPS), we consider two cases: (1) network worstcase delay is smaller than the control sampling period; (2) network worst-case delay is larger than the control sampling period.

For the first case, the delivery ratio ( $D R$, defined as the ratio of arrived messages and sent messages) is the key effect on control system performance. To achieve high DR, the network configuration can be set to "as reliable as possible," that is, a high level of redundancy, requiring more nodes. More nodes typically induce more network delay (more traffic on the network), but for this first case, all messages still arrive within the control sampling period and thus the delay has little (if any) effect on the WCS performance.

However, when the worst-case delay is larger than the control sampling period, there is a trade-off between network delay and packet loss for the WCS performance. This more complex case is our focus in this paper. Although the network-induced imperfection problem has been studied from the control perspective $[17,24,27,33]$, minimizing the network-induced imperfections has not been studied from the network perspective. We use network configuration to achieve the trade-off between packet losses (requiring more redundant nodes) and delays (calling for fewer nodes).

This problem can be thought as an optimization problem to find the network configuration with minimum network imperfections, which can be solved offline. However, plant conditions typically change with time and space $[1,6]$, such as electromagnetic and radio frequency interference (EMI/RFI) and moving people/obstacles, which may severely impact the WCS performance. We focus on addressing the time-correlated interference in the network and one of our contributions is an online network reconfiguration scheme.

We consider link failures and define average link success ratio (LSR) as the probability a message can be sent out successfully on that link; we use average LSR as the indication of the average network interference. We solve the problem in two parts, offline 


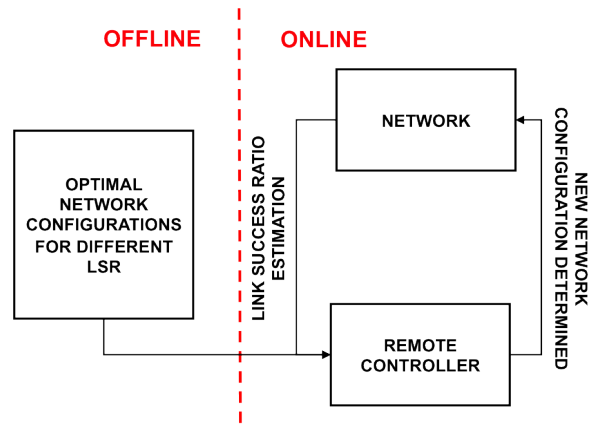

Figure 1: Network reconfiguration for control system with dynamic Interference

and online, as shown in Figure 1. For the offline part, to quantify the network imperfection, we propose a model to transform network delay and delivery ratio into a total induced delay on the control system. We create an optimal estimated network configuration set indexed by LSR values offline, and store it at the controller node. For the online part, at runtime, the controller computes the estimated LSR is and selects a configuration for the network, from the ones computed offline. The controller then broadcasts the new network configuration to all the nodes in the network to carry out a reconfiguration. To study the interaction between network reconfiguration and the WCS, we conduct a case study with a 12-hop and up to 50-node network for a nonlinear primary heat exchanger system in a small modular nuclear reactor in a NPP. The results show that our network imperfection model is accurate and that our network reconfiguration algorithms perform better than a state-of-the-art scheme.

The contributions of this paper encompass:

- the design and implementation of a novel offline and online algorithms for time-correlated link failures

- a network imperfection model that formulates total induced delay in the WCS

- six online reconfiguration algorithms for the WCS

- a case study that presents the in-depth interaction between the network reconfiguration and control

\section{RELATED WORK}

Network-induced imperfections are great challenges for WCSs. The solutions for delay and loss in WCSs can be classified into control only, network only, and control+network co-design solutions.

Control-only solutions for dealing with network imperfections are promising. The authors in [17] model the closed-loop system as a discrete-time switched system and select the optimal stabilizing gain to guarantee the system stability and performance, considering both network time delays and packet losses. Other examples include $[24,27,33]$ that use the model-based predictive control (MPC) approach to address the network imperfections. A state machine as part of MPC, a nonlinear MPC with a Network Delay Compensation (NDC) strategy and output and state predictions are proposed in [24], [27] and [33], respectively. However, these works do not consider network reconfiguration in WCSs.
For the network-only solutions, network reconfiguration is essential, because interference is unpredictable and time-varying. Interference can cause network disconnections, degrading the WCS performance. Different works focus on different network reconfiguration layers. For the data link layer, an online dynamic link layer scheduling algorithm [8] meets the deadline of a rhythmic flow and minimize the number of dropped regular packets, based on a rhythmic task model [11]. TDMA-ASAP adds adaptive slot stealing scheme to TDMA to reduce network delay [4]. For the routing layer, IDDR [39] forwards packets with high integrity requirement to the next hop with smaller queue length and packets with larger weights to choose shorter paths for low delay. Topology control is another active research area to dynamically tolerant node $[18,20,22]$ and link failures [10, 23, 28]. To tolerate node failure, distributed and centralized heuristics algorithms (CORP [14] and SpiderWeb [32]) propose federate the disjoint network segments with least relay nodes and eventually improve the network reliability. To tolerate link failure, $[19,21]$ mitigate the impact of lossy links by maintaining K-connectivity of the network. However, all aforementioned works do not consider WCS performance during reconfiguration (only from the network perspective) and do not conduct case studies, which is an important evaluation step in WCSs.

Integration and co-design of network and control system are effective for WCSs both when exploring network topological conditions and control system stability [25] or the integration of wireless network and control in NPPs [34, 36, 37]. In [16], the author proposes an algorithm on data link layer TDMA scheduling to achieve higher delivery ratio for emergency packets than regular packets. A case study of a wireless-controlled water tank is conducted, but does not address network delay imperfection, since they assume the worst-case network delay is less than the control sampling period. In [3], the authors explore the problem of sharing a wireless channel between multiple control loops and design a control-aware random access communication policy to not violate the stability of any of the control loops. In contrast with the works above, we do not assume the network environment is stable or the network interference is at a given, fixed level.

\section{OFFLINE OPTIMAL NETWORK CONFIGURATION}

We first introduce a model describing the network-induced imperfection impact on the control system performance as caused by the packet loss and network delay. We then use this model to find the set of possible optimal network configurations.

As in [16], we assume sensors do not fail (only consider transient wireless link failure) and when a message is not received by the controller, the previously-received value is used. Our imperfection model is appropriate for tracking and regulation control problems for slowly varying systems with low-pass characteristics [?].

\subsection{Network Imperfection Model}

We define the delay induced into the control system by the wireless network as $T_{\text {used }}-T_{\text {sensed }}$, where $T_{\text {used }}$ is the time the measurement signal is used by the controller and $T_{\text {sensed }}$ is the time the sensor sends out the sensor measurement. We model the delay induced into the control system by 
Dynamic Wireless Network Reconfiguration for Control System applied to a Nuclear Reactor Case Study

$$
D=\left\lceil\frac{D_{\text {network }}+n_{\text {loss }} \Delta_{n s p}}{\Delta_{c s p}}\right\rceil \Delta_{c s p}
$$

where $D_{\text {network }}$ is the network end-to-end delay, $\Delta_{c s p}$ is the control sampling period, $n_{\text {loss }}$ is the number of consecutive packet losses, $\Delta_{n s p}$ is the network sampling period (i.e., the interval between two consecutive sensor readings and/or transmissions). For example, in Figure 2, the $\Delta_{c s p}=\Delta_{n s p}=0.1 s, D_{\text {network }}=0.2 s$; when measurement $M_{2}$ gets lost, the induced delay $D_{2}$ is $0.3 \mathrm{~s}$ and the controller will use measurement $M_{1}$. If $M_{3}$ also gets lost, the induced delay $D_{3}$ becomes $0.4 \mathrm{~s}$ and the controller will (re-)use $M_{1}$.

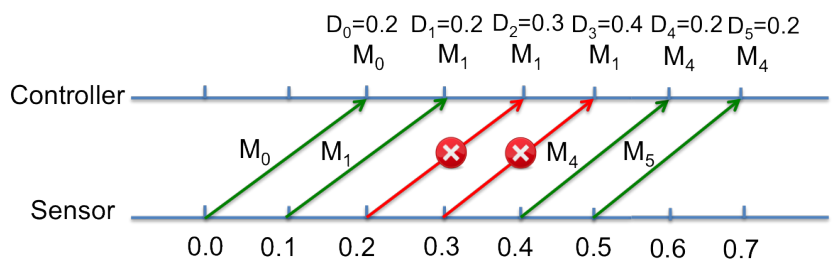

Figure 2: Network delay and loss illustration, when network delay is greater than control sampling period.

$D$ is related to both network delay and the number of consecutive packet losses, which is a function of the packet delivery ratio (delivery ratio estimation and end-to-end worst-case delay estimation have been studied elsewhere $[29,34]) . n_{\text {loss }}$ is estimated by the expected value of the network loss ratio (1-DR). Since DR can be viewed as the probability a message received by the controller, $n_{\text {loss }}=\sum_{i=1}^{n} i(1-D R)^{i} D R,\left((1-D R)^{i} D R \geq c\right)$, where $(1-D R)^{i} D R$ is the probability of $i$ consecutive losses $(i$ consecutive losses and $(i+1)^{t h}$ message received). When the probability is less than a threshold $(c)^{1}$, we assume that the probability can be ignored to avoid long-running computations. For example, for $D R=0.9$ and $c=0.0009$, the probability of getting 1,2 and 3 consecutive losses are $0.09,0.009$, and 0.0009 , respectively. Therefore, the expected number of consecutive losses is $1 \times(1-0.9) \times 0.9+2 \times(1-0.9)^{2} \times 0.9+3 \times(1-0.9)^{3} \times 0.9=0.1107$ The situations of 4 or more consecutive losses are ignored, since the probability of 4 consecutive message losses is $(1-0.9)^{4} \times 0.9=$ $0.00009<c$.

\subsection{Optimal Network Configurations}

Our offline algorithm discovers the set of network configurations that will be saved and used later during the online portion. Since our goal is to minimize the network impact (network delay and packet loss) on the control system, we propose an offline algorithm to find the set of network configurations with minimum induced delay, $D$, considering the network imperfection model in Section 3.1. The steps are as follows. (1) for each average LSR value $(L S R=$ $0.5,0.51,0.52, \ldots, 1.0)$, we estimate the delay induced delay $D$ for each network configuration by applying the worst-case network delay $\left(D_{\text {net work }}\right)$ estimation (e.g., $\left.[29,30,34]\right)$ and DR estimation

\footnotetext{
${ }^{1}$ The value of $c$ is set by users. The less value of $c$, the more accurate, but it takes more time to compute $n_{\text {loss }}$
}

RTNS '18, October 10-12, 2018, Chasseneuil-du-Poitou, France

(e.g., [34]); (2) our algorithm searches all network configurations in a brute-force way (1,500 possibilities: 30 configurations and 50 LSR values) and finds the one with minimum induced delay, $D$; (3) we find a set of estimated optimal network topologies with minimum total induced delay $D$ for each LSR value and store them in a lookup table $T$ indexed by LSR value. Note that our algorithm is generic when we can estimate $D$ for each configuration (how to estimate $D$ is not the focus of this paper).

\section{ONLINE NETWORK RECONFIGURATION}

Given that static configurations do not adapt to the time-varying noise and interference that can cause time-correlated link failures (i.e., the average link quality remains constant over a period of time), we devised an online dynamic network reconfiguration to improve the control system performance and minimize the total induced delay $D$. We assume that the network conditions do not change as fast as the execution of network reconfiguration algorithms and that nodes are time synchronized.

The controller carries out both the control and network reconfiguration algorithms, given that it has all the information needed to decide the optimal configuration. When a reconfiguration is needed (for example, due to interference/noise), the controller propagates a new network configuration to all nodes. To deal with packet reordering, we discard old packets at the remote controller. In this paper, due to space constraints, we only discuss changing the number of sensor nodes for network configuration, even though our offline algorithm (Section 3) is general and can deal with variables like data link layer schedule, network routing, topology, etc. To save network energy consumption (i.e., to prolong network lifetime), active nodes are put to sleep if not needed and other nodes are activated when needed, creating different network topologies. The synchronization of the network topology is done through actuation messages in reserved slots, as in many proposed TDMA algorithms (e.g., [38]). Our online network reconfiguration is based on the look-up table $T$ computed offline (see previous Section) given the current LSR. Below, we first propose an algorithm to estimate LSR at run time. We then propose six online network reconfiguration algorithms, three considering consecutive packet losses (Section 4.3) and three not (Section 4.2).

\subsection{Network Average LSR Estimation}

To estimate the LSR at run time, we propose a jumping window in-network aggregation method. During a certain LSR estimation interval (LSRI), each node counts the number of messages received, $n_{r e c}$, and the number of messages it should receive, $n_{\text {should }}$ (knowing the period of message arrival). At the end of the LSRI, each node concatenates the two numbers, $n_{\text {rec }}$ and $n_{\text {should }}$ to its message, and sends the message to its parent nodes, which will sum their own $n_{r e c}$ and $n_{\text {should }}$ with their children's $n_{r e c}$ and $n_{\text {should }}$ values, respectively; this repeats until getting to the controller. Eventually, the remote controller will compute the final overall network average LSR by its received $n_{r e c}$ and $n_{\text {should }}$ as $\frac{n_{\text {rec }}}{n_{\text {should }}}$. Algorithm 1 shows the LSR estimation algorithm running on one relay node in more detail. Note that our algorithm is general and can be applied to any kind of network topology. 


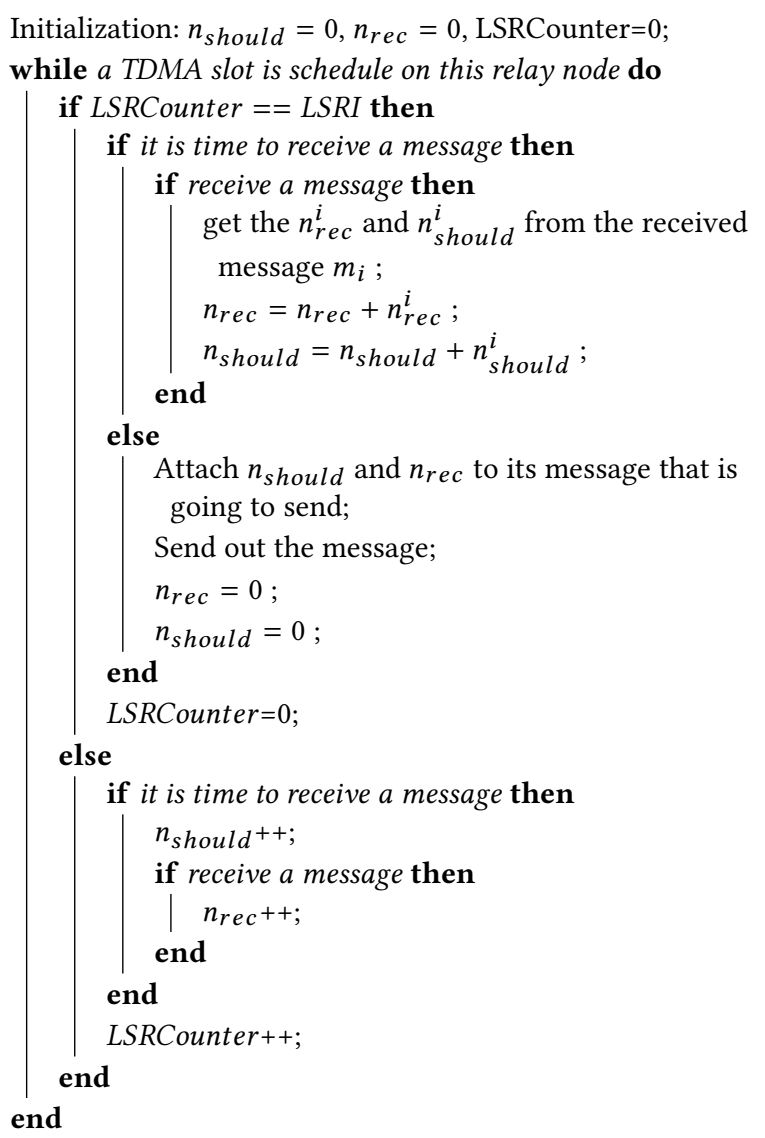

Algorithm 1: LSR estimation algorithm on one active relay node

\subsection{Reconfiguration Not Considering Consecutive Losses}

When the LSR changes, we extract the optimal configuration from Table $T$. We explore three options to reach the optimal configuration, namely DirectJump to Optimal (DO), Multiplicative Increase and Conservative Decrease (MICD), and Adaptive Control (AC). We will consider consecutive message losses in Section 4.3.

4.2.1 DO (Direct Jump). Adjust the network topology to instantaneously have the exact number of nodes that correspond to the optimal network topology.

4.2.2 MICD (Multiplicative Increase and Conservative Decrease). Inspired by [31], with a focus on network reliability, the number of nodes is multiplicatively (i.e., very quickly) increased when needed (converse of TCP/IP protocols window reduction [2]). When the current number of nodes is more than the estimated optimal number of nodes, the number of nodes is conservatively decreased (in our case, we reduce by 1 node).

4.2.3 AC (Adaptive Control). Inspired by adaptive control theory [9], the larger the difference between the estimated optimal number of nodes (est node $)$ and the current number of nodes ( curr $_{\text {node }}$ ) is, the faster we add or remove nodes. That is, curr $_{\text {node }}=\alpha \times$ curr $_{\text {node }}+(1-\alpha) \times e^{2} t_{\text {node }}$, rounded to the closest integer. We use

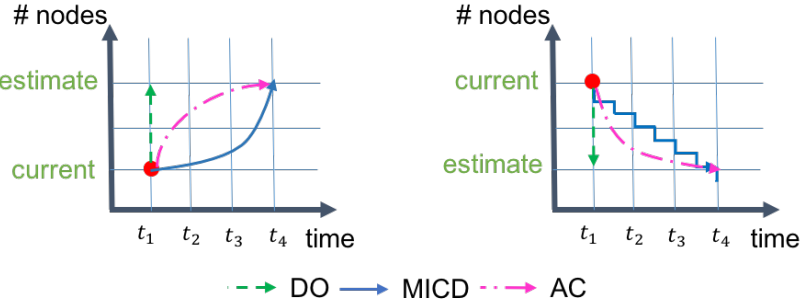

Figure 3: Comparison of online algorithms; increase (left) and decrease (right) of nodes

a parameter $\alpha$ that guides the speed of addition and reduction of nodes in the network $(0<\alpha<1)$. When $\alpha=0$, AC behaves like $\mathrm{DO}$ and the speed to add or reduce nodes is maximum. When $\alpha=1$, the current number of nodes does not change, that is, it is a static network. In essence, smaller $\alpha$ implies higher speed to change the current number of nodes.

Figure 3 illustrates the difference among the algorithms. When the estimated number of nodes is greater than the current number of nodes (left part of Figure 3), DO will directly jump to the estimated number. MICD exponentially increases the number of nodes to make sure the network is reliable. AC will add the number of nodes fast at first, then slowly approaching to the estimated number. When the current number of nodes is greater than the estimated number of nodes (right part of Figure 3), DO will also directly jump to the estimated number. But MICD will conservatively decrease the number of nodes by 1 at a time to make sure the network remains reliable. AC will decrease the number of nodes fast at first, and slowly approach to the estimated number.

\subsection{Online Reconfiguration Considering Consecutive Losses}

From Equation 1, the total induced delay is proportional to the number of consecutive losses $n_{\text {loss }}$. However, in algorithms in Section 4.2 , we did not consider $n_{\text {loss }}$. Since the LSR estimation is not completely accurate, there could be consecutive message losses, which will degrade the control system performance. In other words, when there are consecutive message losses, we need to make the network is more robust (we choose to add more nodes). As a first experimental step, whenever $n_{\text {loss }} \geq m$, we add $k$ more nodes in the network. $m$ and $k$ are user-selected parameters; we use $m=k=3$; how to tune the parameters is not the focus of this paper.

Considering consecutive losses, we devise three more online algorithms called CL-DO, CL-MICD, and CL-AC.

\section{PRIMARY HEAT EXCHANGER CASE STUDY}

We conducted a case study to show and experiment with our wireless network reconfiguration for a nonlinear primary heat exchanger system (PHX) in a NPP [5]. Safety issues in NPPs are beyond the scope of this paper; we focus on feasibility first.

The PHX has its main function the exchange of heat from inside of the reactor to the outside. The PHX is typically modeled as a nonlinear system, and a nuclear reactor model typically has three PHXs. For simplicity of the presentation, we only discuss wireless 
Dynamic Wireless Network Reconfiguration for Control System applied to a Nuclear Reactor Case Study

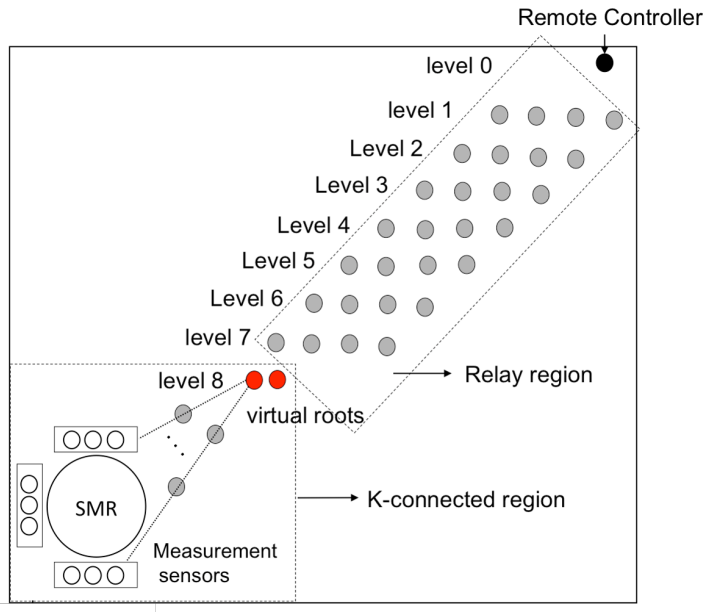

Figure 4: Network topology design

control for one PHX. Three measurements are periodically sent to the remote controller, namely outlet hot leg temperature, inlet hot leg temperature, and mass flow rate. In this case study, we only consider the wireless communication from measurement sensors to the remote controller. Possible solutions for the round trip communication could be TDMA scheduling analysis [7, 29] or different routing schemes[16].

We deploy a 12-hop and up to 50 nodes network in the NPP. We wake up some inactive nodes and put some active nodes to sleep, as reconfigurations are needed. As shown in Figure 4, the network contains two parts: a $k$-connected region and a relay region (we only show $k=2$ ). We connect these two regions by virtual roots. Messages go from sensors to virtual roots, and then to the controller. The routing in each region is done differently.

In the $k$-connected region, there are $k$ edge-disjoint paths (here we consider $k \leq 4$ ) from the sensors to the virtual roots. The number of paths is set depending on the current network link quality. The more edge-disjoint paths are required, the more nodes need to be active in the network and the higher the DR. In this paper, we activate paths from left to right (deactivate in the opposite direction). Experimentally, the direction of path activation showed no significant difference.

In the relay region, there is a line of primary nodes and at most three lines of backup nodes from the virtual roots to the remote controller. In this paper, we activate backup nodes from the highest level to the lowest level for each line of backup nodes Each time we activate as many backup nodes as needed (e.g., if our algorithm in Sect 4 decides to add 10 backup nodes, one and a half line of backup nodes is added). We have experimented with adding nodes from lowest to highest level and random, without significant differences in the results (Section 6.2.5). Note that we use backup nodes/paths, instead of multi-channel is because adding backup nodes is still necessary when the network interference noise is very high and simple sensors do not support multi-channel transceivers.

We use the bitvector protocol [35], which uses TDMA scheduling to guarantee real-time transmission, as follows. In bitvector, relay
RTNS '18, October 10-12, 2018, Chasseneuil-du-Poitou, France

nodes are organized in a children-parent relationship (as shown in Figure 4). In the $k$-connected region, messages are sent via the multiple edge-disjoint paths. Note that the virtual roots can receive duplicate messages from multiple paths, but only send out one message with the corresponding measurement (drop the duplicate messages by overhearing its siblings' messages). Therefore, the more number of paths, the more reliable the network. In the relay region, relay nodes broadcast messages level by level towards the controller. Within each level, the primary node will broadcast first, then the first, second, and third backup nodes, in order. If the backup parent finds out (while overhearing and checking the bit vector) that the primary did not send out the values, the backup parent will send out the message. Therefore, the more active nodes in the network, the higher DR and also the network delay. The worst-case network delay is $n \Delta t$, where $n$ is the number of the current active nodes and $\Delta t$ is the time slot of TDMA scheduling $(\Delta=10 \mathrm{~ms}$ [16] in our case study ).

\section{QUANTITATIVE RESULTS}

We combined the implementations of bitvector protocol with a state-of-the-art cyber-physical system simulator (WCPS 2.0 [16]) and allow wireless network to run together with the primary heat exchanger system simulink model. The online reconfiguration algorithms (see Section 4) are implemented on the controller node.

To simulate time-varying link failures, we propose a network fault model as follows. We hold RSSI (received signal strength [13]) constant for a period of time (based on real-life noise traces [16]) and change RSSI to another value for the next period of time (the duration is based on the noise traces mentioned above). We adjust each relay node's RSSI to change LSR within the range $(0.5,1.0)$, depending on the following three quantities: RSSI duration: the time interval at which the RSSI is fixed (after that, the RSSI may be changed); RSSI range and time range: the value and time range the RSSI duration is chosen from. We randomly choose RSSI from RSSI range with a uniform distribution and randomly choose RSSI duration from time range also with a uniform distribution. Figure 5 shows an example of the generated RSSI values varying over time using our fault model with RSSI range of $(-60 \mathrm{dBm},-85 \mathrm{dBm})$ and time range of $(0,20 \mathrm{~s})$.

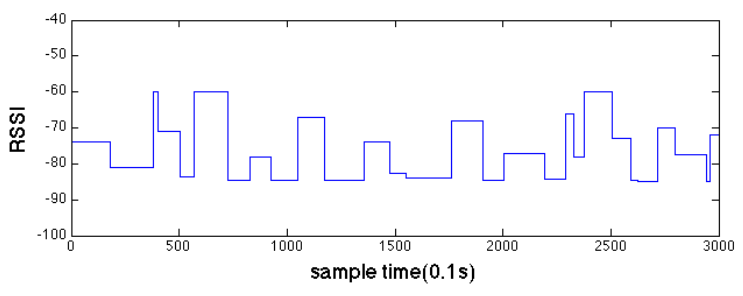

Figure 5: Time-correlated RSSI variation example

To study the behavior of the PHX, we consider the case when an operator changes the power output for the reactor. To evaluate the performance of the control system, we adopted three metrics: Integral Absolute Error (IAE) [15], Maximum Absolute Error (MAE) [15] and Root Mean Square Error (RMSE), which is the RMS error in the power output of the PHX measured between the closed-loop 
Table 1: Simulation Parameters and Values

\begin{tabular}{|c|c|}
\hline Parameters & Values \\
\hline Network sampling period $\left(\Delta_{n s p}\right)$ & $0.1 \mathrm{~s}$ \\
\hline Control sampling period $\left(\Delta_{n s p}\right)$ & $0.1 \mathrm{~s}$ \\
\hline Simulation time & $300 \mathrm{~s}$ \\
\hline LSRI Range & $2 \mathrm{~s}, 4 \mathrm{~s}, 8 \mathrm{~s}, 12,16 \mathrm{~s}, 20 \mathrm{~s}$ \\
\hline Reference functions & ramp30, ramp60, ., ramp120 \\
\hline$\alpha$ value & $0.1,0.2, \ldots, 0.9$ \\
\hline RSSI values & {$[-60 \mathrm{dBm},-85 \mathrm{dBm}]$} \\
\hline
\end{tabular}

responses using wired control (i.e., no packet drops and no network delay) and wireless control under consideration. These metrics quantify the quality of the WCS: the less (error), the better. We only show IAE and RMSE results in the paper, given that the MAE result is very similar. We also measure the induced delay (to analyze IAE, MAE and RMSE) of the network imperfection model, the number of nodes that are used in the network, and the network lifetime. Table 1 shows our simulation parameters and values.

\subsection{Offline Optimal Network Configuration}

We apply the delivery ratio (DR) and worst-case end-to-end delay estimation in [34] to calculate the estimated total delay $D$ induced into the PHX. By applying the algorithm in Section 3.2, we can get the look-up table containing the optimal configuration, as shown in Figure 6 (left), the estimated optimal number of nodes for different LSR values. The higher the LSR, the higher the DR, and the more robust the network will be, and therefore the fewer sensor nodes needed. The optimal number is always a multiple of 10 and the total induced delay is a multiple of $0.1 \mathrm{~s}(\Delta \operatorname{csp})$ due to the ceiling operation in our network imperfection model (see Equation 1). For example, a network with 25 active nodes (network delay is $0.25 \mathrm{~s}$, but is considered as $0.3 \mathrm{~s}$ due to the ceiling operation) will definitely induce more IAE in the control system than the network with 30 active nodes (network delay is also $0.3 \mathrm{~s}$ ), since 30 nodes is more reliable than 25 .

To see the correlation of the network imperfection model from Section 3.1 and the control system performance, we run the simulation with static RSSI values. Figure 6 (middle) shows the induced delay for different number of nodes and different RSSI values. Note that when the number of nodes is 20 , the network is not robust and has more consecutive message losses, thus has more induced delay even though the actual network delay is the lowest (for the messages that are actually delivered), since it has the smallest number of nodes. When the number of nodes is 50 , the opposite behavior occurs.

Figure 6 (right) shows the power output RMSE of the PHX. Comparing the middle and right parts of Figure 6, we can see that our network imperfection model is accurate visually; it is also statistically accurate (Pearson correlation $r=0.993, p<0.001$ ) correlating well to the power output RMSE.

\subsection{Online Network Reconfiguration Results}

To simulate time-correlated link quality models, we fine-tuned the RSSI range and duration to get different representative network fault models with different average RSSI values. We simulate our system on five fault models with average RSSI values (in $\mathrm{dBm}$ ) of $-65,-70,-74,-78$ and -82 In this section, we present control system performance and network lifetime results for different online reconfiguration algorithms.

6.2.1 Heat exchanger system power reference function. We change the power reference function (i.e., the required power output of a nuclear reactor), to reduce power from $42 \mathrm{MW}$ to $37.8 \mathrm{MW}$ (typical values for NPPs) in an interval of time; for example, Ramp30 means that the power reduction should take 30 seconds. As shown in Figure 8, the steeper the reference function, the larger the IAE: a steep reference function requires a more aggressive power output reduction, which causes more transient responses, which causes larger IAE. Thus, our online algorithms are most useful when there is little time for adjustment (e.g., quick correction of the power output) or when the difference in power is large (e.g., at startup of a new reactor when other reactors have to adjust their power output). Still, our algorithms are also effective under all circumstances; while our worst scenario is for ramp120, the cumulative gains of our algorithms (i.e., the difference in error we provide) are still significant: $14 \mathrm{MW}$ over a 5-minute interval (when RSSI $=-82$ ). Note that the online network reconfiguration algorithms perform similarly for all reference functions. We only present the results for ramp30 to save space.

6.2.2 Comparison of Online Reconfiguration Algorithms. Figure 9 (top) shows the power output IAE of the PHX for different average values of RSSI and different online network reconfiguration algorithms; RMSE and MAE results are similar to the IAE results and are thus omitted. In the figure, "best static" is the best fixed number of nodes in the network, as follows. We tested the number of nodes 20 to 50 for each fault model and chose the static scheme with minimum average IAE over all the fault models (in our case it is static scheme with 40 active nodes). For the first study, we add 3 more nodes $(k=3)$ in the $\mathrm{CL}^{-}{ }^{*}$ algorithms when 3 consecutive message losses $(m=3)$ happen. As the average RSSI value increases, the power output IAE increases, since the network has more interference. As expected, our dynamic algorithms perform typically better than the static scheme for all fault models. CL-DO and CL-AC algorithms perform better than the other dynamic algorithms, because they add more nodes only when needed, that is, when the network has consecutive message losses. Figure 10 shows the comparison over 3,000 samples of CL-MICD and CL-AC in 20 experiments. The reason CL-MICD always performs worse among CL algorithms is because the speed of reducing the number of nodes is slow (reduce one at a time) and the speed of adding nodes is fast (exponential increase), which often overshoots the number of actually needed nodes and thus causes more induced delay (induced delay of CL-MICD is always higher than CL-AC) and degrades the control system performance.

We calculate the average network lifetime to measure the network energy consumption. We define our network lifetime as the average sensor node lifetime, and calculate the average network energy consumption during one round of measurements of transmissions from sensor to the controller. For simplicity, we assume a general battery capacity is $8640 \mathrm{~J}$, which is the typical capacity of two AA batteries. Figure 9 (middle) shows the network lifetime for 

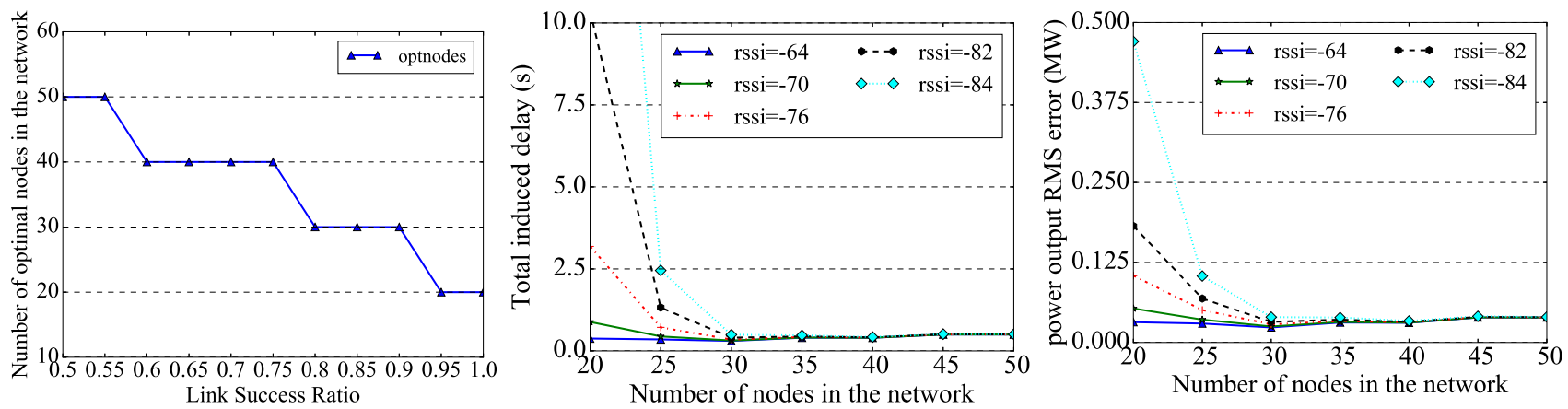

Figure 6: Offline look-up table: estimated optimal network configuration of different LSR values (left); total induced delay result for $R S S I=-64$ to $R S S I=-84$ that correspond to average $L S R$ values of $0.93,0.88,0.82,0.77$, and 0.72 , respectively (middle); power output RMSE against a network with no error no delay, that is, wired control system (right)

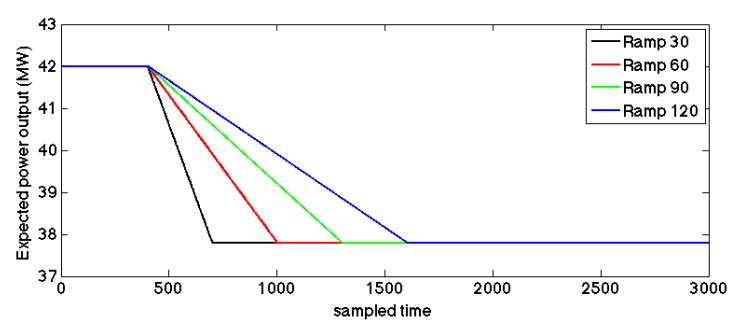

Figure 7: Control system power reference functions

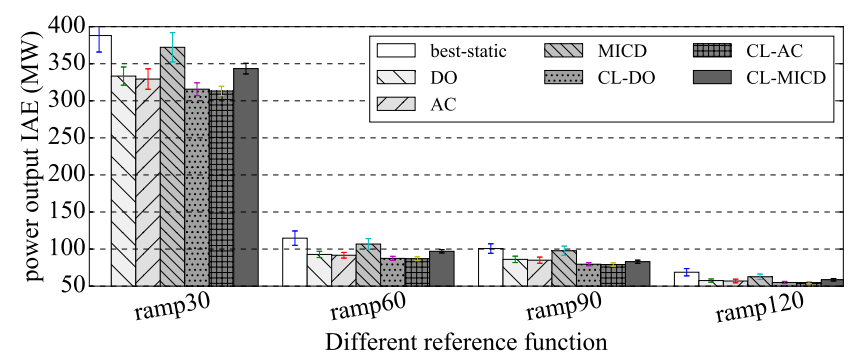

Figure 8: Power output IAE for different reference functions (average $\mathrm{RSSI}=-82 \mathrm{dBm}$ and LSRI $=2$ s (20 samples))

different reconfiguration algorithms. Algorithms considering consecutive message losses (CL-DO, CL-AC and CL-MICD) consume more energy than their counterparts not considering consecutive message losses (DO, AC and MICD). This is because CL-* algorithms are more aggressive adding additional nodes when there are consecutive losses. In addition, when there is more interference in the network, the network consumes more energy, since the network needs more backup nodes to handle link failures. For network performance results, see Figure 17 in Appendix. As the average RSSI value decreases, indicating more interference in the network environment, the DR decreases, but the network delay increases since more nodes are reconfigured participating.
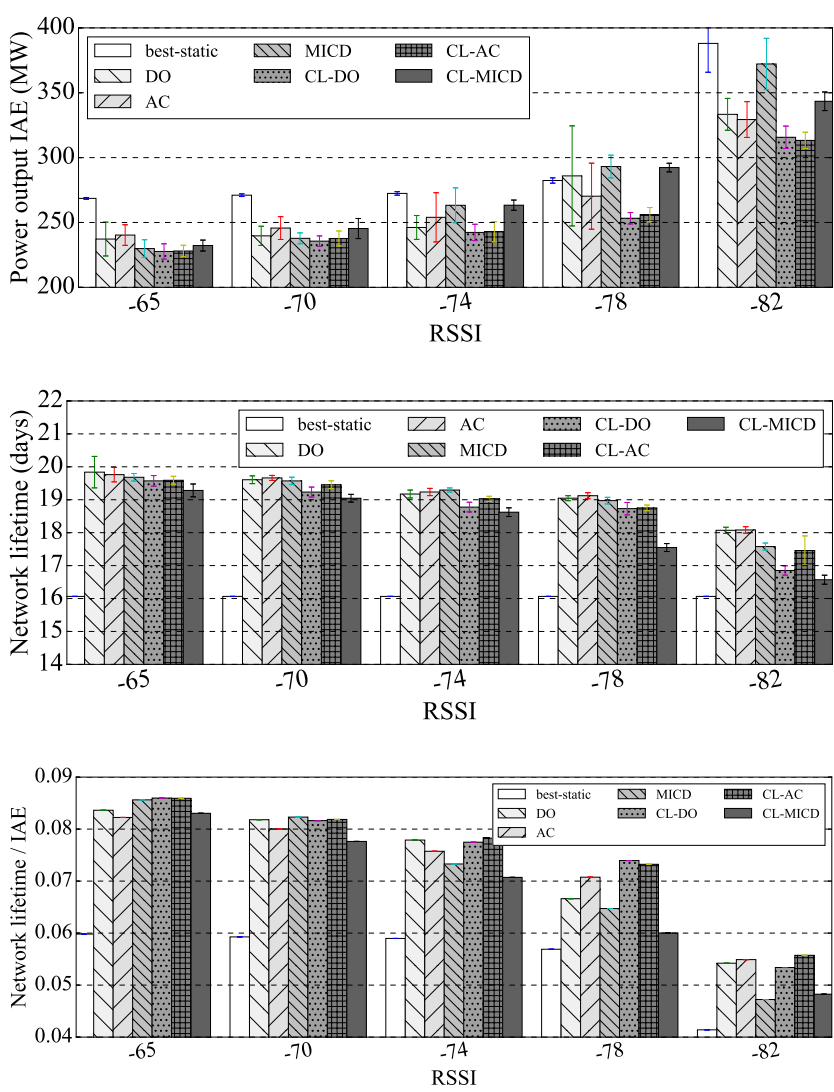

Figure 9: Power output IAE (top), network lifetime (bottom) and network lifetime / IAE results for different RSSI values (LSRI: $2 s ; \alpha$ : 0.1)

To consider both control system performance and network energy consumption together, we normalize network lifetime by IAE in Figure 9 (bottom) for different average RSSI values. The static 


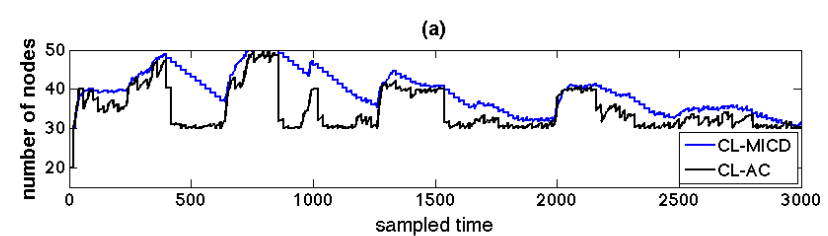

(b)

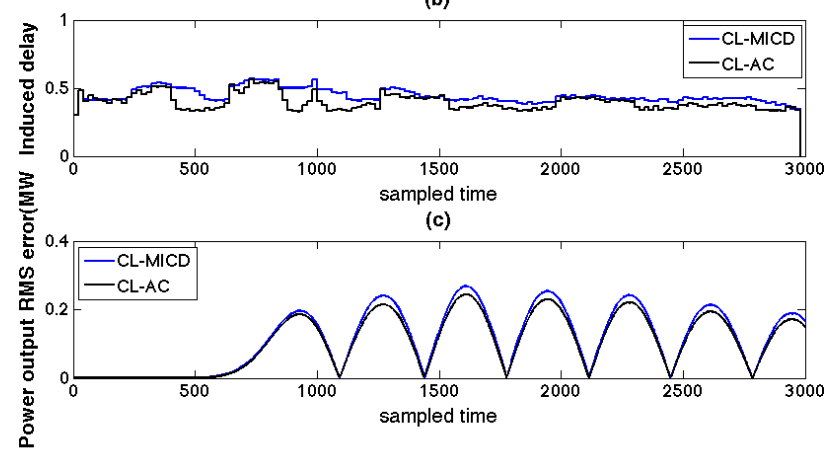

Figure 10: (a) Average number of nodes in the network, (b) average induced delay and (c) average RMSE over time (LSRI: 2s; Average RSSI: $-82 \mathrm{dBm}$; CL-AC algorithms with $\alpha$ : 0.1;)

scheme is significantly worse than the dynamic algorithms, because it consumes the most network energy consumption, and has the highest IAE, demonstrating that our reconfiguration algorithms are necessary and work well. Note that we selected the best (minimum IAE) static scheme to be conservative in our evaluation, but in reality it would be hard to select a good static configuration a priori, since the network interference is unpredictable. CL-DO and CL-AC perform similarly over all fault models and always perform better than the other online reconfiguration algorithms.

6.2.3 Sensitivity Analysis of LSR Estimation Interval. Since LSR is estimated periodically, the length of the LSR estimation interval (LSRI) will affect the control system performance. Figure 11 (top) shows that When the LSRI increases, the IAE of algorithms DO, MICD and AC increase because our estimation is less accurate at high LSRI values. The middle and bottom figures of Figure 11 show the network lifetime and network lifetime normalized by IAE, respectively. For network performance (network DR and delay), see Figure 18 in Appendix. In Figure 12, the green line is the real LSR; the black line (LSRI of 2s) tracks the real LSR better than the LSRIs of $8 \mathrm{~s}$ and 16s. Therefore, the control system performance has less error when the LSR estimation is more accurate. Figure 13 shows the comparison of the DO with LSRI of 2 s and 20s (AC and MICD has similar results). From sample 600 to 800 , the DO with LSRI of 20 s runs with 30 nodes in the network because the LSR is estimated high averaged over the last 200 samples (from 400 to 600). However, from sample 600 to 800 , the LSR is low (network has more interference) and 30 nodes cannot handle the link failures, which causes consecutive message losses (induced delay $D$ is high) and negatively affects the control system performance. The IAEs of the CL-" algorithms are not affected by the LSRI values because, even though the LSR estimation may not be accurate, $\mathrm{CL}^{-}{ }^{*}$ algorithms
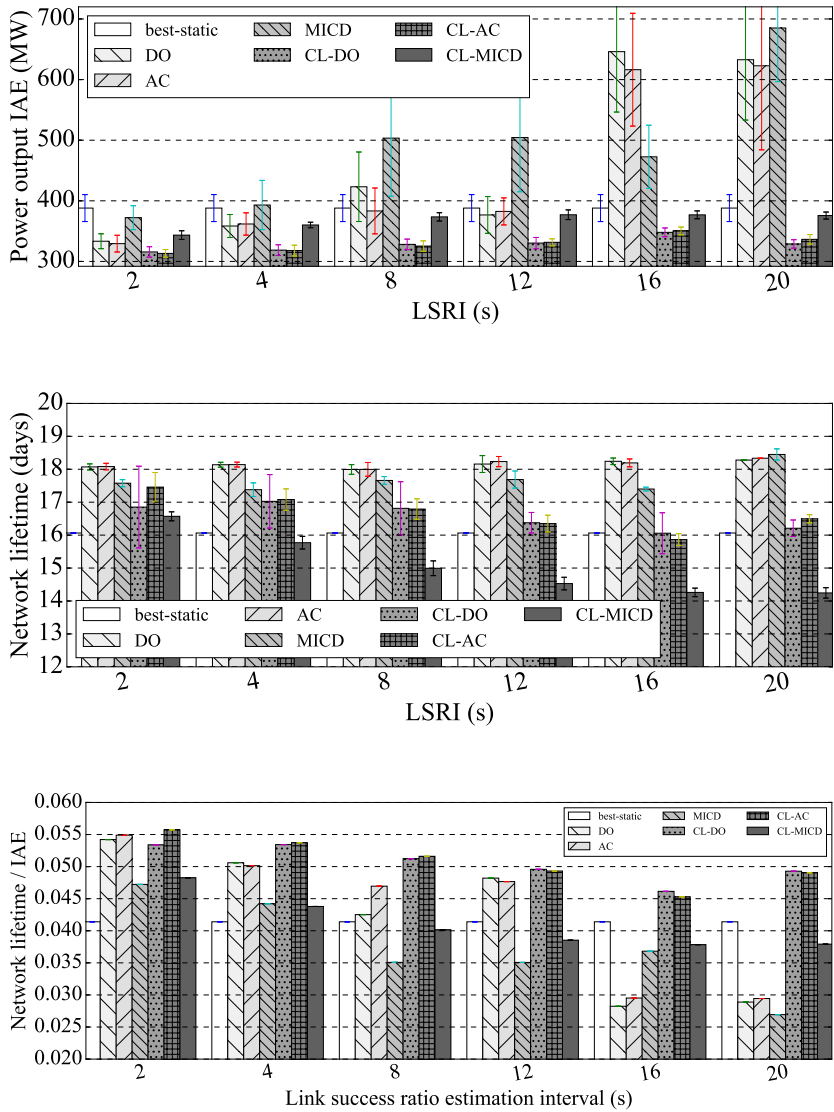

Figure 11: Power output IAE (top), network lifetime (middle) and network lifetime / IAE results for different LSRIs (bottom) for different LSRIs (RSSI: $-82 \mathrm{dBm} ; \alpha: 0.1$ )

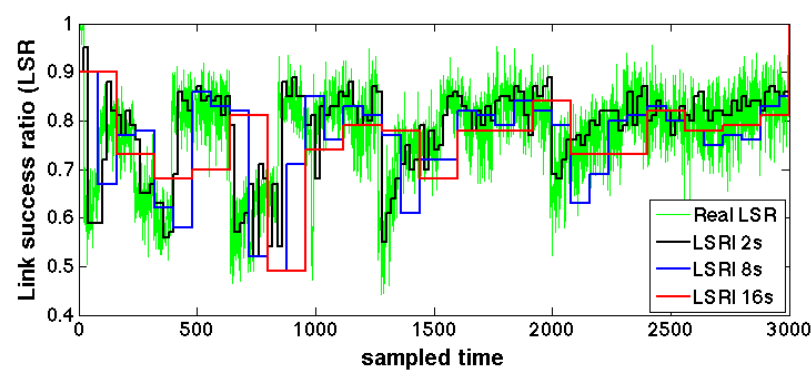

Figure 12: Comparison of estimated and real LSRs (average RSSI $=-\mathbf{8 2} \mathrm{dBm}$ )

add additional nodes to compensate to make the network robust. However, the side-affect is that $\mathrm{CL}^{-}{ }^{*}$ algorithms consume more energy. CL-DO and CL-AC perform better than the other online reconfiguration algorithms over different LSRI values. 
Dynamic Wireless Network Reconfiguration for Control System applied to a Nuclear Reactor Case Study
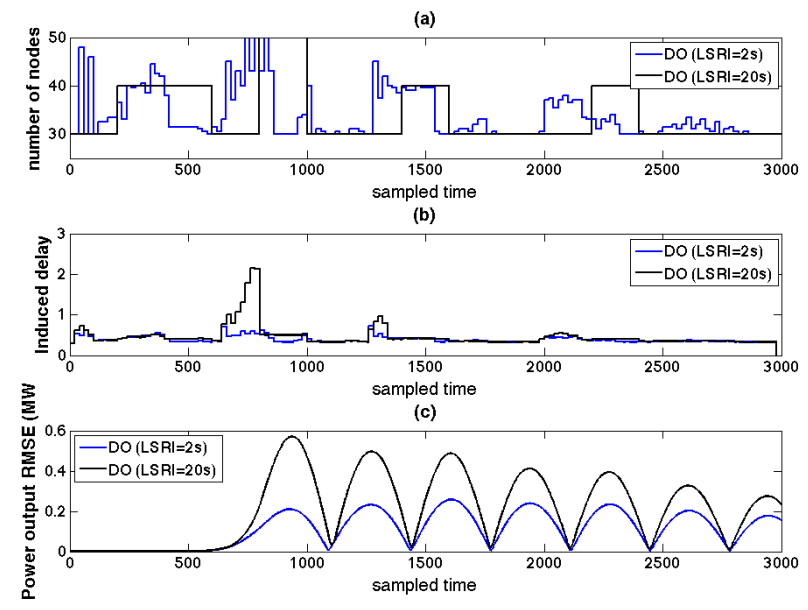

Figure 13: (a) Average number of nodes in the network, (b) average induced delay and (c) average RMSE over time (LSRI: 2s; Average RSSI value: $-82 \mathrm{dBm}$;)

6.2.4 Adaptive control algorithm with different $\alpha$ values. Recall that the AC algorithm has a variable alpha $(0.1 \leq \alpha \leq 0.9)$, which determines the speed to add or reduce nodes in the network (small $\alpha$, fast node adding). The $\alpha$ value can also affect the control system performance. Figure 14 shows the IAE of AC and CL-AC algorithms for different $\alpha$ values. First, for AC, when $\alpha<0.5$, the control system performs the same. This is because when the speed of adding or removing nodes is slow $(\alpha>0.5)$ it cannot react to the LSR variation fast enough. Figure 15 shows the reason more clearly. From sample 600 to 800 , when the network has more interference, the speed of AC $(\alpha=0.9)$ of adding nodes is slower than the AC $(\alpha=0.1)$, causing consecutive message losses and more induced delay. From sample 800 to 1300 , when the network has less interference, the speed of $\mathrm{AC}(\alpha=0.9)$ of reducing nodes is also slow and induce more delay (network delay is high) into the control system.

Figure 14 also shows that CL-AC always performs better than AC. Although the speed to add or reduce nodes is slow for $\alpha=0.9$, considering consecutive losses can compensate somewhat when the network has more interference. Figure 15 shows more details. From sample 600 to 800 , when the network has more interference (consecutive message losses happen), CL-AC ( $\alpha=0.9)$ adds more nodes in the network than $\mathrm{AC}(\alpha=0.9)$, which improves control system performance. But CL-AC may add more nodes than needed. From sample 1300 to 1600 , CL-AC $(\alpha=0.9)$ adds too many nodes and causes more induced delay (see Figure 15 (b)) and thus consumes more network energy when compared with $\mathrm{AC}(\alpha=0.9)$.

6.2.5 Comparison of Nodes Activation Methods. For nodes activation methods, we activated nodes from the highest level to the lowest level (deactivating nodes in the opposite direction: from the lowest to the highest level), from the lowest level to the highest level, or randomly in the relay region; activating paths from left to right, right to left and randomly in k-connected region. Figure 16 shows that different activation methods in the relay region do not
RTNS '18, October 10-12, 2018, Chasseneuil-du-Poitou, France

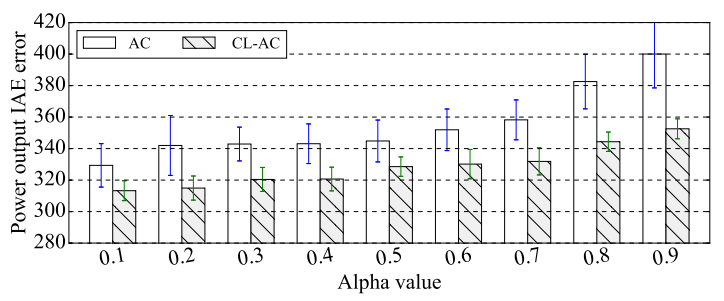

Figure 14: Power output IAE result comparison of AC and CL-AC for different alpha values (RSSI value: -82dBm; LSRI: 2s)
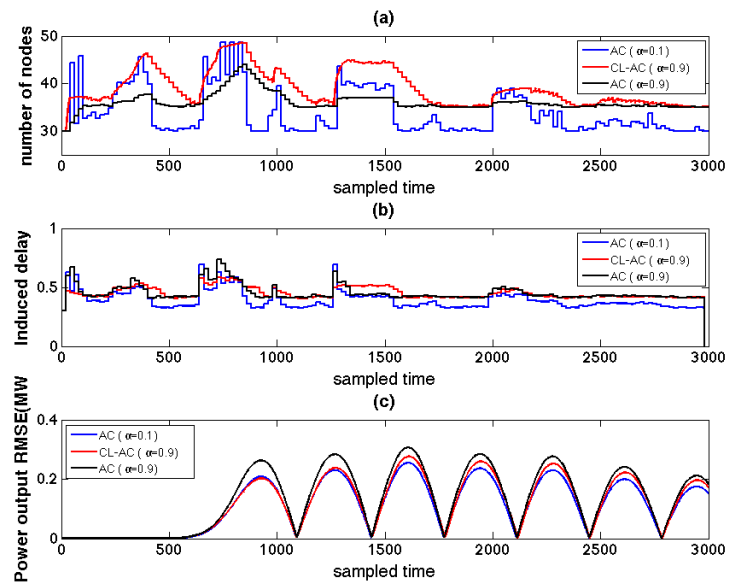

Figure 15: (a) Average number of nodes in the network, (b) average induced delay and (c) average RMSE over time for AC $(\alpha=0.1)$, CL-AC $(\alpha=0.9)$ and AC $(\alpha=0.9)$ (average RSSI value: -82dBm; LSRI: $2 s$;)

make significant impact on control system performance and energy consumption. The three methods also behave very similarly for DR and network delay (not shown). The activation methods comparison in k-connected region also shows no significant difference (not shown).

\section{CONCLUSION AND FUTURE WORK}

In recent years, wireless control systems (WCSs) over a multi-hop network has been well explored. However, the interaction between network reconfiguration and control, when the worst-case network delay is bigger than the control sampling period has not been researched. In this paper, we propose and implement a WCS framework with offline and online parts. We also propose a network imperfection model and network configuration determination estimation offline; we then propose one online LSR estimation algorithm and six online network topology control algorithms with and without considering consecutive losses. We also conduct a case study to see the in-depth interaction between network reconfiguration and the control. Our simulation result shows that our network imperfection model is accurate with Pearson correlation 

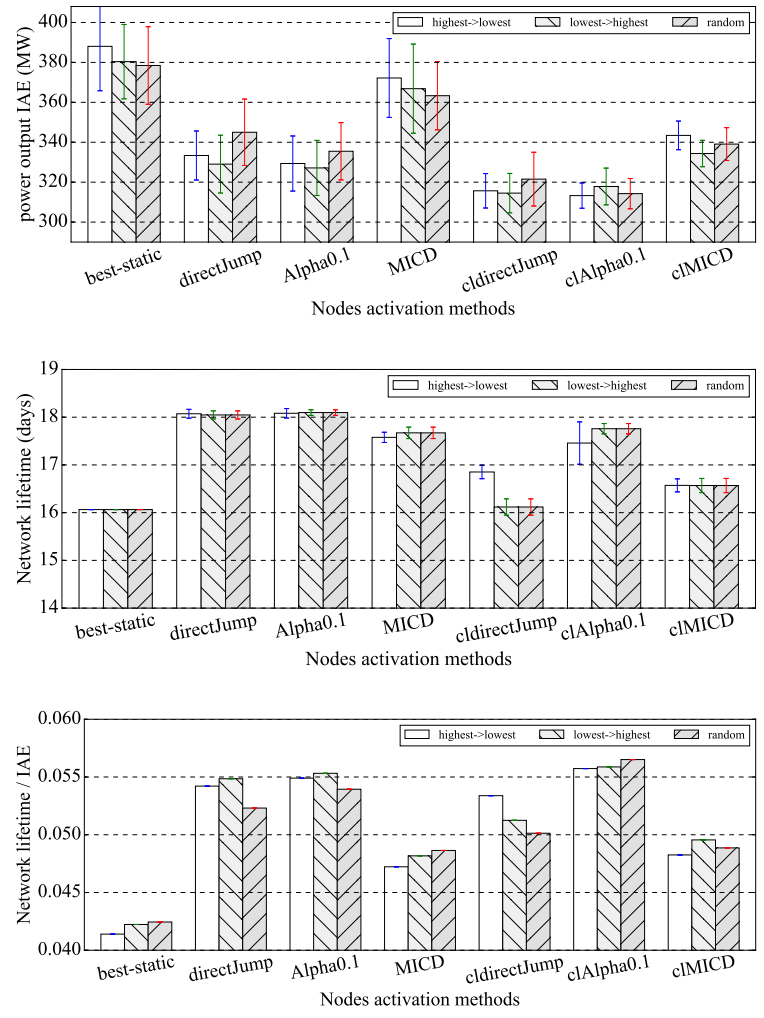

Figure 16: (a) Power output IAE result, (b) network lifetime results and (c) Network lifetime / RMSE results for different adding/reducing nodes methods (the average RSSI value: -82 dBm; AC and CL-AC algorithms with $\alpha$ : 0.1; LSRI: 2 s; activation method in k-connected region is from left to right)

0.993, that network reconfiguration works better than the stateof-the-art static scheme showing low error and longer network lifetime. We find that considering consecutive message losses can improve the control system performance due to more robustness in the network. We also found that online reconfiguration schemes, CL-DO and CL-AC, perform better than the others in reducing the network-induced error and saving network energy consumption.

In the future, we will explore other network aspects, such as routing layer and data link layer reconfiguration.

\section{A NETWORK DELIVERY RATIO AND DELAY}

Figures 17 and 18 show the network delivery ratio and delay for different average RSSI values and LSRIs, respectively. (a)

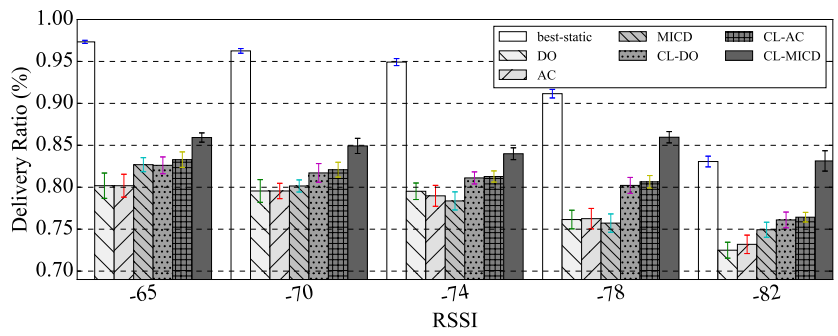

(b)

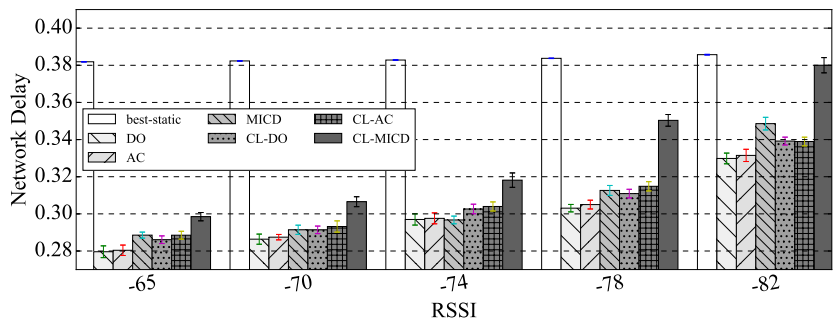

Figure 17: (a) Network delivery ratio and (b) network delay for different average RSSI values

(a)

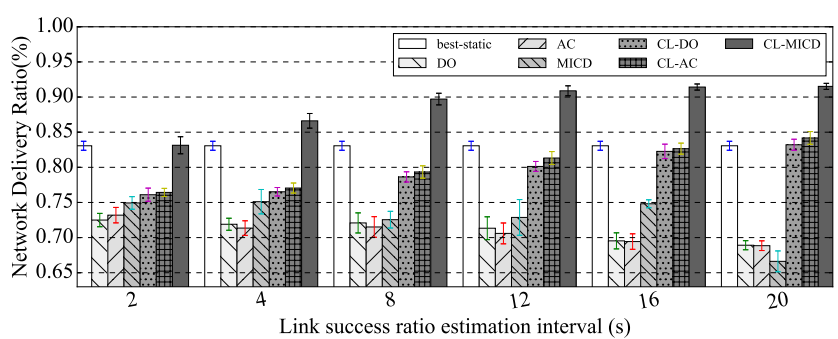

(b)

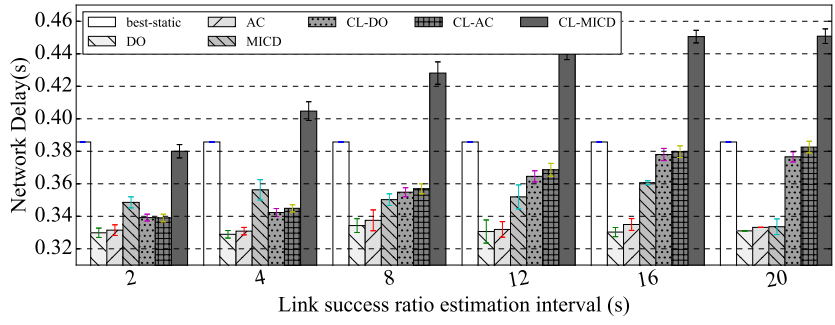

Figure 18: (a) Network delivery ratio and (b) Network delay for different LSRIs(the average RSSI value: $-82 \mathrm{dBm}$; AC and CL-AC algorithms with $\alpha=0.1$ )

\section{REFERENCES}

[1] N. Baccour, A. Koubâa, L. Mottola, M. A. Zúñiga, H. Youssef, C. A. Boano, and M. Alves. Radio link quality estimation in wireless sensor networks: a survey. ACM Trans on Sensor Networks, 8(4):34, 2012. 
Dynamic Wireless Network Reconfiguration for Control System applied to a Nuclear Reactor Case Study

[2] D.-M. Chiu and R. Jain. Analysis of the increase and decrease algorithms for congestion avoidance in computer networks. Computer Networks and ISDN systems, 17(1):1-14, 1989.

[3] K. Gatsis, A. Ribeiro, and G. J. Pappas. Control-aware random access communication. In 2016 ACM/IEEE 7th International Conference on Cyber-Physical Systems (ICCPS), pages 1-9. IEEE, 2016.

[4] S. Gobriel, D. Mosse, and R. Cleric. Tdma-asap: Sensor network tdma scheduling with adaptive slot-stealing and parallelism. In Int'l Conf on Distributed Computing Systems (ICDCS) 2009., pages 458-465, 2009.

[5] S. R. Greene, J. C. Gehin, D. E. Holcomb, J. J. Carbajo, D. Ilas, A. T. Cisneros, V. K. Varma, W. R. Corwin, D. F. Wilson, G. L. Yoder Jr, et al. Pre-conceptual design of a fluoride-salt-cooled small modular advanced high-temperature reactor (smahtr) Oak Ridge National Laboratory, Oak Ridge, TN, Report No. ORNL/TM-2010/199, Fig, pages $8-1,2010$

[6] V. C. Gungor, B. Lu, and G. P. Hancke. Opportunities and challenges of wireless sensor networks in smart grid. IEEE transactions on industrial electronics, 57(10):3557-3564, 2010.

[7] S. Han, X. Zhu, A. Mok, D. Chen, and M. Nixon. Reliable and real-time communication in industrial wireless mesh networks. In 17th IEEE Real-Time and Embedded Technology and Applications Symp, 2011.

[8] S. Hong, X. S. Hu, T. Gong, and S. Han. On-line data link layer scheduling in wireless networked control systems. In 2015 27th Euromicro Conference on Real-Time Systems, pages 57-66. IEEE, 2015.

[9] N. Hovakimyan and C. Cao. Adaptive Control Theory: Guaranteed Robustness with Fast Adaptation. SIAM, 2010.

[10] V. Kawadia and P. Kumar. Power control and clustering in ad hoc networks. In INFOCOM 2003, volume 1, pages 459-469. IEEE, 2003

[11] J. Kim, K. Lakshmanan, and R. R. Rajkumar. Rhythmic tasks: A new task model with continually varying periods for cyber-physical systems. In Int't Conference on Cyber-Physical Systems, pages 55-64, 2012.

[12] K.-D. Kim and P. Kumar. The importance, design and implementation of a middleware for networked control systems. In Networked Control Systems, pages 1-29. Springer, 2010.

[13] H. Lee, A. Cerpa, and P. Levis. Improving wireless simulation through noise modeling. In 2007 th International Symposium on Information Processing in Sensor Networks, pages 21-30. IEEE, 2007.

[14] S. Lee and M. Younis. Optimized relay placement to federate segments in wireless sensor networks. IEEE fournal on Selected Areas in Communications, 28(5):742-752, 2010.

[15] B. Li, Y. Ma, T. Westenbroek, C. Wu, H. Gonzalez, and C. Lu. Wireless routing and control: a cyber-physical case study. In ACM/IEEE International Conference on Cyber-Physical Systems, 2016.

[16] B. Li, L. Nie, C. Wu, H. Gonzalez, and C. Lu. Incorporating emergency alarms in reliable wireless process control. In Int'l Conference on Cyber-Physical Systems, pages 218-227. ACM, 2015.

[17] H. Li, M.-Y. Chow, and Z. Sun. Optimal stabilizing gain selection for networked control systems with time delays and packet losses. IEEE Transactions on Control Systems Technology, 17(5):1154-1162, 2009.

[18] L. Li, J. Y. Halpern, P. Bahl, Y.-M. Wang, and R. Wattenhofer. Analysis of a conebased distributed topology control algorithm for wireless multi-hop networks. In ACM symposium on Principles of distributed computing, pages 264-273. ACM, 2001.

[19] N. Li and J. C. Hou. Flss: a fault-tolerant topology control algorithm for wireless networks. In Proceedings of the 10th annual international conference on Mobile computing and networking, pages 275-286. ACM, 2004.

[20] N. Li, J. C. Hou, and L. Sha. Design and analysis of an mst-based topology control algorithm. IEEE Transactions on Wireless Communications, 4(3):1195-1206, 2005.

[21] X.-Y. Li, P.-J. Wan, Y. Wang, and C.-W. Yi. Fault tolerant deployment and topology control in wireless networks. In Proceedings of the 4th ACM international symposium on Mobile ad hoc networking \& computing, pages 117-128. ACM, 2003.

[22] E. L. Lloyd, R. Liu, M. V. Marathe, R. Ramanathan, and S. Ravi. Algorithmic aspects of topology control problems for ad hoc networks. Mobile Networks and applications, 10(1-2):19-34, 2005.

[23] S. Narayanaswamy, V. Kawadia, R. S. Sreenivas, and P. Kumar. Power control in ad-hoc networks: Theory, architecture, algorithm and implementation of the compow protocol. In European Wireless Conference, pages 156-162, 2002.

[24] A. Onat, T. Naskali, E. Parlakay, and O. Mutluer. Control over imperfect networks: Model-based predictive networked control systems. IEEE Transactions on Industrial Electronics, 58(3):905-913, 2011.

[25] M. Pajic, S. Sundaram, G. J. Pappas, and R. Mangharam. Topological conditions for wireless control networks. In 2011 50th IEEE Conference on Decision and Control and European Control Conference, pages 2353-2360. IEEE, 2011.

[26] M. Pajic, S. Sundaram, G. J. Pappas, and R. Mangharam. The wireless control network: A new approach for control over networks. IEEE Transactions on Automatic Control, 56(10):2305-2318, 2011.

[27] G. Pin and T. Parisini. Networked predictive control of uncertain constrained nonlinear systems: recursive feasibility and input-to-state stability analysis. IEEE Transactions on Automatic Control, 56(1):72-87, 2011.
RTNS '18, October 10-12, 2018, Chasseneuil-du-Poitou, France

[28] R. Ramanathan and R. Rosales-Hain. Topology control of multihop wireless networks using transmit power adjustment. In INFOCOM 2000. Nineteenth Annual Joint Conference of the IEEE Computer and Communications Societies. Proceedings. IEEE, volume 2, pages 404-413. IEEE, 2000.

[29] A. Saifullah, D. Gunatilaka, P. Tiwari, M. Sha, C. Lu, B. Li, C. Wu, and Y. Chen. Schedulability analysis under graph routing in wirelesshart networks. In RealTime Systems Symposium, 2015 IEEE, pages 165-174. IEEE, 2015.

[30] A. Saifullah, Y. Xu, C. Lu, and Y. Chen. End-to-end delay analysis for fixed priority scheduling in wirelesshart networks. In Real-Time and Embedded Technology and Applications Symposium (RTAS), 2011 17th IEEE, pages 13-22. IEEE, 2011.

[31] Y. Sankarasubramaniam, O. B. Akan, and I. F. Akyildiz. Esrt: event-to-sink reliable transport in wireless sensor networks. In Proceedings of the 4th ACM international symposium on Mobile ad hoc networking \& computing, pages 177-188. ACM, 2003.

[32] F. Senel, M. F. Younis, and K. Akkaya. Bio-inspired relay node placement heuristics for repairing damaged wireless sensor networks. IEEE Transactions on Vehicular Technology, 60(4):1835-1848, 2011.

[33] A. Ulusoy, O. Gurbuz, and A. Onat. Wireless model-based predictive networked control system over cooperative wireless network. IEEE Transactions on Industrial Informatics, 7(1):41-51, 2011.

[34] W. Wang, C. D’Angelo, D. Mosse, and D. Cole. Integrating control and faulttolerant wireless network design for small modular nuclear reactors. In Information Reuse and Integration (IRI), 2016 IEEE 17th International Conference on, pages 332-342, 2016.

[35] W. Wang, D. Mosse, and D. G. Cole. Bitvector: Fault tolerant aggregation scheme for monitoring in nuclear power plants. In ICESS 2015.

[36] W. Wang, D. Mosse, J. G. Pickel, and D. Cole. Work-in-progress: Cross-layer real-time scheduling for wireless control system. In Real-Time and Embedded Technology and Applications Symposium (RTAS), 2017 IEEE, pages 149-152. IEEE, 2017.

[37] W. Wang, D. Mosse, J. G. Pickel, and D. Cole. Work-in-progress: Wireless network reconfiguration for control systems. In Real-Time and Embedded Technology and Applications Symposium (RTAS), 2017 IEEE, pages 145-148. IEEE, 2017.

[38] J. Yackovich, D. Mosse, A. Rowe, and R. Rajkumar. Making wsn tdma practical: Stealing slots up and down the tree. In Embedded and Real-Time Computing Systems and Applications (RTCSA), 2011 IEEE 17th International Conference on, volume 1, pages 41-50. IEEE, 2011.

[39] J. Zhang, F. Ren, S. Gao, H. Yang, and C. Lin. Dynamic routing for data integrity and delay differentiated services in wireless sensor networks. IEEE Transactions on Mobile Computing, 14(2):328-343, 2015.

[40] L. Zhang, H. Gao, and O. Kaynak. Network-induced constraints in networked control systemsâĂŤa survey. IEEE Transactions on Industrial Informatics, 9(1):403$416,2013$. 